CASE REPORT

\title{
Acute Pain Management in a Child: A Case Report of 46-Days of Popliteal Sciatic Nerve Catheter
}

\author{
Hertz Laurent*, Sola Chrystelle, De La Arena Pablo and Dadure Christophe \\ Department of Anesthesia and Critical Care Medicine, Lapeyronie University Hospital, Montpellier, France

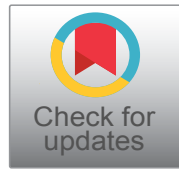

*Corresponding author: Hertz Laurent, MD, Department of Anesthesia and Critical Care Medicine, Lapeyronie University Hospital, Avenue Gaston Giraud 34000, Montpellier, France, Tel: +33788014415, E-mail: I-hertz@chu-montpellier.fr

\begin{abstract}
Background: The use of perineural catheter for more than 72 hours is rare in perioperative practice, but was especially reported for chronic pain, oncologic related pain or palliative care. The main concern remains the risk of neurological or infectious complication. No guideline clearly specifies the maximum duration of perineural catheter maintenance and the safety of long-term catheters is discussed.
\end{abstract}

Case report: We described the case of an 11-year-old boy who suffered a serious injury on his foot. An ultrasound guided sciatic nerve block was performed, with placement of a non-tunneled perineural catheter. The catheter could be used for 46 days with an excellent efficiency both for analgesia (continuous infusion) and anesthesia (additional bolus for surgical procedure). No infectious or neurological related complication to regional anesthesia was notified.

Conclusion: This case demonstrates all the benefits that can be expected by long-term perineural catheter, even if it should be managed with great caution and after careful assessment of the risk-benefit balance.

\section{Introduction}

With the development of ultrasound (US) in regional anesthesia (RA), the safety of peripheral nerve block catheters (PNBC) has been recently confirmed in pediatric anesthesia [1]. However, as in adults, there are no recommendation on the maximum acceptable duration of PNBC placement in children. The main concern on the use of long-term PNBC remains the risk of neurological or infectious complications. Recently, the rate of infectious complications related to PNBC was 0.9\% (95\% $\mathrm{Cl}=0.5-1.4 \%$ ) [2]. The only factor associated with greater incidence of catheter-related infection, was the total number of days before removal of the catheter with an increased risk after 3 days of catheter maintenance [2]. But only few transient and no major neurologic complications were reported [2]. In pediatric, PNBC are often used for postoperative analgesia after orthopedic or general surgery and are typically removed after only 2-3 days. Long term used of PNBC have yet been described for control of chronic pain, oncologic related pain or palliative care in young adults and children [3-5]. We present the case of a child who required the use of a PNBC over a long period of 46 days for perioperative pain management. The child and his family consented to the anonymous publication of this case.

\section{Description of the Case}

An 11-years-old boy, weighting $38 \mathrm{~kg}$, without medical or surgical history, was admitted to our unit after a motor vehicle-pedestrian trauma. The child presented a faciocranial trauma with an initial loss of consciousness but a Glasgow score of 14 at the arrival of medical assistance. A fracture of the right horizontal branch of the mandibular corpus was diagnosed and treated by surgical osteosynthesis. Moreover, there was a severe injury with a large soft tissue defect extending from the internal malleolus to the second phalangeal of the right hallux and musculotendinous and osseous exposure. No vascular compromise was noted and the limited initial neurological exam was not able to objectify significant nerve injury or deficit. The patient complained of diffuse pain. However, shocked by the accident, it was not possible to assess the level of his pain. It was decided to treat him by sedation-analgesia with ketamine. Surgical 
debridement was performed a few hours after the accident under general anesthesia. Given the importance of the injury and the loss of substance, the expected level of pain was significant. Therefore, at the same time, an US-guided continuous sciatic nerve block was performed in popliteal location. Patient controlled regional analgesia was performed using a basal continuous infusion of $2 \mathrm{~mL} / \mathrm{hr}$. of $0.2 \%$ Ropivacaïne (Ropivacaïne ${ }^{\circledR}$, Fresenius Kabi, Sevres, France) and additional boluses of $2 \mathrm{ml}$ with 20 min lockout interval. Efficacy was immediately appreciated by the child to the permanent control of his pain and for the necessary daily cares in the unit. The clinical supervision of the child whose pain, as well as the monitoring of the catheter were performed every 6 hours by the nurses of the service.

The child's care resulted in 56 days of hospitalization in our department until he was transferred to a rehabilitation center. Multiple surgical procedures were required for wound surgical treatments and orthopedic management: Dressings, cleaning, flap (D8), skin grafts (D18 and D36), and cuneo-metatarsal articulation external fixation (D33). An additional injection, ranging from 50 to $100 \mathrm{mg}$ of Mepivacaïne $10 \mathrm{mg} / \mathrm{mL}$ (Carbocaïne ${ }^{\circledR}$, B Braun, Melsungen, Deutschland) or 20 to $50 \mathrm{mg}$ of Ropivacaïne (Concentration of $2 \mathrm{mg} / \mathrm{mL}$ or $3.3 \mathrm{mg} / \mathrm{mL}$ ) through the PNBC, allowed to perform all of these invasive surgical procedures without general anesthesia requirement or use of morphinomimetic molecule. To the patient's request, light sedation, ketamine and/or Propofol infusion, was occasionally used to ensure comfort and anxiety control. Before catheter suppression, at the $46^{\text {th }}$ day, an ultrasound exam was done and showed the proper placement of the catheter, in extraneural situation but close to the nerve structure. No visible abnormality of nervous structure and no local sign of infection or inflammation have been noticed. In terms of general signs, there were only two febrile episodes during hospitalization, both after dressings, with no sustained fever or any other clinical signs of sepsis. The bacteriological study of the perioperative bone tissue found an Enterococcus faecium and an Enterobacter cloacae, but all haemocultures remained negatives. With respect to the resistance patterns of the bacteria found, the child received amoxicillin/clavulanic-acid for 10 days followed by a bi-antibiotic treatment with vancomycin-ciprofloxacin, and finally by vancomycin-tazocillin for a total of 28 days. Biologically, there was a steady decline in inflammatory syndrome during hospitalization. Inflammation blood markers remained normal after the decline of the initial biological inflammatory syndrome.

At the last appointment, on the $119^{\text {th }}$ day of treatment, the child reported symptoms of neuropathic pain in the form of electrical discharges. These were well controlled by gabapentin (Pfizer, New York, USA). Otherwise, in concordance with the expected sequelae, motor testing was still disturbed especially on the adductor, abductor and flexor hallucis muscles but seemed to improve. An irreducible claw of the toes and sensitive deficit of the plantar face of the hallux as well as a hypoesthesia of the dorsal face of the hallux were described. Slight support on the limb was possible using single crutch. In total, the PNBC remained in place for 46 days without infectious or neurological complication, with both continuous and intermittent use, for iterative anesthesia as well as for long-term perioperative analgesia.

\section{Discussion}

We report the safety and efficiency of a long-term peripheral nerve block catheter in context of repeated surgical procedures and perioperative pain management.

The results of the Pediatric Regional Anesthesia Network suggested that maintaining a PNBC could increase the risk of infection [2]. Although no cases of abscess or systemic infection were reported, the total number of days before catheter removal was the only factor associated with a higher incidence of infection [2]. The risk is then increased after 3 days of maintenance [2]. This can lead to systematic withdrawal. However, previous pediatrics studies have qualified this 3-days rule, both in the surgical setting [6], and for chronic or cancer pain control [7]. Obviously, the maintenance of a PNBC should be evaluated according to the benefit-risk ratio specific to each patient and medical issue. This principle of risk-benefit balance was fundamental in this case: While no inflammatory or infectious signs appeared, analgesia was consistently effective and avoided the repetition of general anesthesia for iterative surgical procedure [8]. Strict supervision of the insertion of catheter and monitor of usual vital signs remains the essential safety element. Systematic monitoring of biological parameters such as CRP could also be important for some authors [9] while other suggest antimicrobial prophylaxis [7]. With the help of these elements, this case suggests greater freedom in the decision to maintain a PNBC.

Another attitude was extensively discussed in our team: The systematic repositioning of a new catheter to reduce the risk of infection. It has been described for central venous catheters with more than 21 days of use but the literature does not provide any evidence for the utility of this preventive practice [10]. The significant risk of losing the benefit of this effective catheter and the risk of nerve damage during repeated needle punctures determined our choice to continue the management of this child with the same catheter.

Although very rare, another theoretical risk was neurological sequelae by inflammation related to the long term presence of foreign bodies close to the nerve structure $[11,12]$. In this case, neurological signs have been linked to the trauma and consequences of the immobilization. This unsystematized symptoms were not characteristic of neuritis. However, the neurological 
assessment was stopped 73 days after the withdrawal of the catheter because the patient was lost of our follow-up.

With ultrasound, the correct extraneural position of the catheter was verified at the time of placement and withdrawal. If the ultrasound in the performance of RA is no longer to be demonstrated [1], this case could also illustrate a possible safety gain obtained by ultrasound allowing the precise placement of catheter in extraneural position and its related extensive efficiency.

Finally, this case brings a good report in the maintenance of long-term PNBC, framed by strict rules of positioning and monitoring. Depending on the location of the catheter, in a more risky area, the safety of longterm catheters could be seriously compromised and should be managed with great caution and after careful assessment of the risk-benefit balance.

\section{Conclusion}

Peripheral nerve block catheters have gained an important place in both post-operative setting and chronic pain unit. This case demonstrates all the benefits that can be expected by PNBC placement: Efficient pain control during and after surgery, important reduction in the use of anesthetic drugs and systemic opioid with concomitant decrease in related side effects and adverse events. In this case a large number of general anesthesia were avoided. No infection or systematic neurological deficit related to regional anesthesia was reported. If the catheter can be managed with great caution and after careful assessment of the risk-benefit balance, the long term maintenance could have its place in the management of the child's pain.

\section{Financial Disclosures}

None.

\section{Conflicts of Interest}

The authors declare no conflict of interest.

\section{Running Head}

This case demonstrates all the benefits that can be expected by continuous nerve block: Efficient pain control, reduction in the use of anesthetic drugs and systemic opioid. No infection or systematic neurological deficit was reported.

\section{References}

1. DKM Lam, GN Corry, BCH Tsui (2016) Evidence for the use of ultrasound imaging in pediatric regional anesthesia: A Systematic Review. Reg Anesth Pain Med 41: 229-241.

2. Walker BJ, Long JB, De Oliveira GS, Szmuk P, Setiawan C, et al. (2015) Peripheral nerve catheters in children: An analysis of safety and practice patterns from the pediatric regional anesthesia network (PRAN). $\mathrm{Br} J$ Anaesth 115: 457-462.

3. G Ivani, L Codipietro, F Gagliardi, F Rosso, V Mossetti, et al. (2003) A long-term continuous infusion via a sciatic catheter in a 3-year-old boy. Paediatr Anaesth 13: 718-721.

4. HL Pacenta, RN Kaddoum, LA Pereiras, EJ Chidiac, LL Burgoyne (2010) Continuous tunnelled femoral nerve block for palliative care of a patient with metastatic osteosarcoma. Anaesth Intensive Care 38: 563-565.

5. Burgoyne LL, Pereiras LA, Bertani LA, Kaddoum RN, Neel $M$, et al. (2012) Long-term use of nerve block catheters in paediatric patients with cancer related pathologic fractures. Anaesth Intensive Care 40: 710-713.

6. Dadure C, Bringuier S, Nicolas F, Bromilow L, Raux O, et al. (2006) Continuous epidural block versus continuous popliteal nerve block for postoperative pain relief after major podiatric surgery in children: A prospective, comparative randomized study. Anesth Analg 102: 744-749.

7. Doralina L Anghelescu, Brittney L Harris, Lane G Faughnan, Linda L Oakes, Kelley B Windsor, et al. (2012) Risk of catheter-associated infection in young hematology/oncology patients receiving long-term peripheral nerve blocks. Paediatr Anaesth 22: 1110-1116.

8. KM Posfay-Barbe, DM Zerr, D Pittet (2008) Infection control in paediatrics. Lancet Infect Dis 8: 19-31.

9. KJ Downes, SL Weiss, JS Gerber, Klieger SB, Fitzgerald JC, et al. (2017) A pragmatic biomarker-driven algorithm to guide antibiotic use in the pediatric intensive care unit: The optimizing antibiotic strategies in sepsis (OASIS) study. $J$ Pediatr Infect Dis Soc 6: 134-141.

10. NP O'Grady, M Alexander, LA Burns, Dellinger EP, Garland J, et al. (2011) Guidelines for the prevention of intravascular catheter-related infections. Am J Infect Control 39: S1-S34.

11. R Brull, CJL McCartney, VWS Chan, H El-Beheiry (2007) Neurological complications after regional anesthesia: Contemporary estimates of risk. Anesth Analg 104: 965-974.

12. BM Ilfeld (2011) Continuous peripheral nerve blocks: A review of the published evidence. Anesth Analg 113: 904925. 